\title{
Dupilumab: A New Paradigm for the Treatment of Allergic Diseases
}

\author{
Sastre J1', Dávila I² \\ 1Allergy Service, Fundación Jiménez Díaz, and Faculty of Medicine, Universidad Autónoma de Madrid, Madrid, Spain \\ Institute for Biomedical Research of Salamanca (IBSAL), Allergy Service, University Hospital of Salamanca, and Department of Biomedical and \\ Diagnostics Sciences, Faculty of Medicine, Salamanca, Spain
}

J Investig Allergol Clin Immunol 2018; Vol. 28(3): 139-150

doi: 10.18176/jiaci.0254

\begin{abstract}
Moderate and severe forms of allergic diseases such as atopic dermatitis and asthma are a challenge for clinicians. In these conditions, which severely affect the quality of life of the patient and frequently have associated allergic comorbidities, the therapeutic options are often very limited. Treatment with systemic corticosteroids and immunosuppressants has adverse effects in the long term, and a significant proportion of patients remain refractory to therapy. In this context, the emerging biological drugs constitute a truly innovative therapeutic approach. A leading example is dupilumab, a monoclonal antibody targeting the $\alpha$ chain of the interleukin (IL)-4 receptor. Dupilumab inhibits the biological effects of the cytokines IL-4 and IL-13, which are key drivers in the $T_{H} 2$ response. The efficacy and safety profile of dupilumab in the treatment of allergic diseases has been tested for more than 10 years in a variety of large clinical trials in atopic dermatitis, asthma, chronic rhinosinusitis with nasal polyposis, and eosinophilic esophagitis. In 2017, the United States Food and Drug Administration and the European Medicines Agency approved the use of dupilumab for the treatment of adult patients with moderateto-severe atopic dermatitis whose disease is not adequately controlled with prescribed topical treatment. The results of phase III clinical studies of dupilumab in patients with persistent, uncontrolled asthma have been highly promising. The safety and tolerability profile of dupilumab has proven to be very favorable in long-term clinical trials. In this review, we focus on the mechanism of action of dupilumab, its development, and its impact on daily clinical practice in allergic diseases.
\end{abstract}

Key words: Allergy. Atopic dermatitis. Asthma. Biological drugs. Dupilumab.

\section{Resumen}

Las formas clínicas moderadas y graves de enfermedades alérgicas comunes como la dermatitis atópica o el asma constituyen un reto para los clínicos. En estos casos, que afectan intensamente la calidad de vida del paciente y con frecuencia conllevan otras enfermedades alérgicas asociadas, las opciones terapéuticas son a menudo muy limitadas. El tratamiento con corticosteroides sistémicos o inmunosupresores tiene efectos adversos a largo plazo y una proporción significativa de pacientes se muestra refractaria a la terapia. En este contexto, los nuevos fármacos biológicos, dirigidos a la base inmunológica de la enfermedad, ofrecen un enfoque terapéutico verdaderamente innovador. Un ejemplo destacado de estos fármacos es dupilumab, un anticuerpo monoclonal dirigido contra la cadena alfa del receptor de la interleucina (IL)-4. Dupilumab inhibe los efectos biológicos de las citoquinas IL-4 e IL-13, unos de los principales efectores de la respuesta Th2. La efectividad y seguridad de dupilumab en el tratamiento de enfermedades alérgicas se han probado durante más de diez años en una variedad de grandes ensayos clínicos en dermatitis atópica, asma, rinosinusitis crónica con poliposis nasal y esofagitis eosinofílica. La FDA y la EMA aprobaron en 2017 el uso de dupilumab en el tratamiento de pacientes adultos con dermatitis atópica moderada o grave que no se controla adecuadamente con tratamiento tópico. Los estudios clínicos de Fase 3 de dupilumab en pacientes con asma persistente no controlada también han sido muy prometedores. En los ensayos clínicos a largo plazo la seguridad y tolerabilidad de dupilumab ha demostrado ser muy elevada. En esta revisión nos hemos centrado en el mecanismo de acción de dupilumab, su desarrollo como fármaco y su impacto en la terapia de enfermedades alérgicas.

Palabras clave: Alergia. Dermatitis atópica. Asma. Medicamentos biológicos. Dupilumab. 


\section{Introduction: Allergic Diseases}

Allergic diseases are characterized by hypersensitivity to specific allergens, which generally leads to increased serum levels of IgE [1-3]. Predisposed individuals typically present 1 or more of a spectrum of interconnected disorders, namely, atopic dermatitis (AD), allergic asthma, allergic rhinitis, food allergy, hymenoptera allergy, or eosinophilic esophagitis (EoE). Allergic diseases are notoriously heterogeneous and result from a complex interaction between genetic and environmental factors [4]. For reasons not fully understood, allergic diseases are increasing in prevalence worldwide. Thus, $\mathrm{AD}$ is one of the most common skin diseases, with prevalences of up to $25 \%$ in children and $2 \%-5 \%$ in adolescents [5]. The estimated worldwide prevalence of AD in adults, where the disease can often be severe, has been estimated at 1\%-3\% [6,7]. In Spain the prevalence of severe AD is $0.08 \%$ [8]. On the other hand, asthma affects $1 \%-18 \%$ of the population depending on the country, or about 300 million people worldwide [2]. The prevalence of allergic rhinitis varies widely by country, but it is estimated that $16 \%$ of adults in Europe could be affected [9]. Chronic rhinosinusitis affects $10.9 \%$ of people in Europe [10], and EoE is increasingly prevalent worldwide (currently $0.4 \%$ in developed countries) [11]. Finally, IgE-mediated food allergy could affect as many as $4 \%-7 \%$ of preschool children and has been increasing in frequency in both developed and developing countries in recent decades $[12,13]$.

Allergic diseases often develop sequentially at different stages of life in the same individuals, possibly because of a shared genetic origin. For example, a recent study of 380 adult $\mathrm{AD}$ patients showed that $40.3 \%$ had asthma, $51.3 \%$ had allergic rhinitis, $24.2 \%$ had allergic conjunctivitis, and $60.5 \%$ had other allergic conditions [14]. In addition, AD in early childhood is strongly associated with asthma later in life [15] and can have a huge impact on the quality of life (QOL) of affected individuals. The secondary effects of the illness can result in profound emotional, psychological, economic, and social burdens for patients and their families [16]. In the case of asthma, although patients with severe forms of the disease represent a minority of the total asthma population, they frequently experience comorbidities and account for an important part of asthma-associated health care costs [17].

Clinically and mechanistically, allergic diseases are characterized by systemic inflammation and type- 2 helper $\mathrm{T}$-cell $\left(\mathrm{T}_{\mathrm{H}} 2\right)$ activation, which in turn is characterized by the production of interleukin (IL) 4, IL-5, IL-9, and IL-13. For example, activation of $\mathrm{T}_{\mathrm{H}} 2$ in the airways leading to production of eosinophilia or IgE occurs in about $50 \%$ of asthmatics [18]. For this reason, recently developed biological therapies for allergic diseases have mainly targeted either the final product, $\operatorname{IgE}[19]$, or the key proximal type-2 cytokines, namely, IL-4, IL-5, and IL-13 [20,21].

Dupilumab is a fully human monoclonal antibody directed against IL-4R $\alpha$, which inhibits IL-4/IL-13 signaling and thus downregulates type-2 immunity. The United States Food and Drug Administration (FDA) approved dupilumab (Regeneron Pharmaceuticals) in March 2017 for "the treatment of adult patients with moderate-to-severe atopic dermatitis whose disease is not adequately controlled with topical prescription therapies or when those therapies are not advisable" [22,23]. In October of the same year it was also approved by the European Medicines Agency (EMA) for "the treatment of moderate-to-severe atopic dermatitis in adult patients who are candidates for systemic therapy" [24]. In addition to AD, dupilumab has proven effective in the treatment of severe and persistent asthma and is being actively investigated for chronic rhinosinusitis with nasal polyposis and for EoE (see below).

In this review, we focus on dupilumab and its mechanism of action, the randomized clinical trials evaluating its efficacy and safety, and its therapeutic value in daily clinical practice for the treatment of $\mathrm{AD}$, asthma, rhinitis, and EoE.

\section{Allergic Diseases: Current Therapeutic Options}

Topical corticosteroids are the main treatment of mild and moderate forms of atopic diseases [1,2]. In the case of $\mathrm{AD}$, topical calcineurin inhibitors (eg, tacrolimus ointment and pimecrolimus cream) and topical phosphodiesterase-4 inhibitors are also approved therapies. Moderate or recurrent forms of $\mathrm{AD}$ may require stronger topical corticosteroids or phototherapy, and treatment of severe forms of AD may require systemic corticosteroids and immunosuppressants such as cyclosporine A, azathioprine, mycophenolate mofetil, enteric-coated mycophenolate sodium, and methotrexate [25]. However, in the most severe cases, treatment options are quickly exhausted owing to significant adverse effects of topical corticosteroids and immunosuppressants in the long term, which often limit treatment [1]. Dupilumab is currently the only biological drug approved for the treatment of moderate-to-severe AD in adults $[23,24]$. Other biological drugs are in phase II clinical trials, namely lebrikizumab (anti-IL-13), tralokinumab (anti-IL-13), nemolizumab (antiIL-31), and tezepelumab (anti-thymic stromal lymphopoietin), although further studies will be required to evaluate their effectiveness (reviewed in [26]).

Asthma is usually treated with inhaled or systemic corticosteroids, alone or in combination with other controller medications such as long-acting $\beta_{2}$-adrenoreceptor agonists (LABAs) and cysteinyl-leukotriene receptor antagonists [2]. Despite the widely available effective therapies for the treatment of asthma, many asthma cases remain uncontrolled [27]. Severe, uncontrolled asthma can lead to corticosteroid dependence, with systemic exposure to these agents potentially leading to serious short- and long-term adverse effects, including weight gain, diabetes, osteoporosis, glaucoma, anxiety, depression, cardiovascular disease, and immunosuppression. Biological therapies approved for asthma include omalizumab (antiIgE), mepolizumab (anti-IL-5), reslizumab (anti-IL-5), and benralizumab (anti-IL-5R $\alpha$ ) [17]. Dupilumab successfully completed 2 large phase III clinical trials in 2017 (see below) [28-33].

Chronic rhinosinusitis is also a complex disease consisting of several variants with specific pathophysiologies [34], and treatment options are also often limited. Current treatments include topical corticosteroids and nasal saline irrigations [35], mometasone implants, and sinus surgery [3]. The biological 
treatments omalizumab (anti-IgE) in the case of comorbid asthma and mepolizumab (anti-IL-5) have been successfully tested. As described below, dupilumab demonstrated efficacy in a phase II clinical trial, and phase 3 clinical trials are currently underway and expected to conclude in 2018.

No specific medications are currently approved by the FDA for treatment of EoE. Standard treatments include proton pump inhibitors, oral corticosteroids, dietary therapy, and dilation [36,37], usually with the objective of interrupting disease progression towards fibrosis. Phase II studies of dupilumab for the treatment of EoE have been completed, and phase III studies are ongoing (see below). Since EoE affects fewer than 200000 people in the US, dupilumab has received orphan status from the FDA for the treatment of this disease [38].

\section{Mechanism of Action of Dupilumab}

Dupilumab is a recombinant human IgG4 antibody to the IL-4 receptor [39]. There are 2 types of IL-4 receptors: the type 1 receptor, which is composed of the IL-4 chain (IL-4R $\alpha$ ) and a $\gamma$ chain $(\gamma \mathrm{C})$, and the type 2 receptor, which is composed of the IL- $4 \mathrm{R} \alpha$ chain and the $\alpha 1$ chain of the IL-13 receptor (IL-13R $\alpha 1$ ) (Figure). These receptors are present in the surface of a large number of cells involved in the pathophysiology of $\mathrm{T}_{\mathrm{H}} 2$ allergic responses, which include B lymphocytes, eosinophils, dendritic cells, monocytes/ macrophages, basophils, keratinocytes, bronchial epithelial cells, endothelial cells, fibroblasts, and airway smooth muscle cells [40]. The type 1 receptor can be activated by IL-4 and the type 2 receptor can be activated by both IL- 4 and IL-13 (Figure). Ligand binding to these receptors activates a signal transduction cascade that mainly leads to the modulation of expression of genes involved in IgE class switching, $\mathrm{T}_{\mathrm{H}} 2$ cell differentiation, and M2 macrophage polarization [21,39].

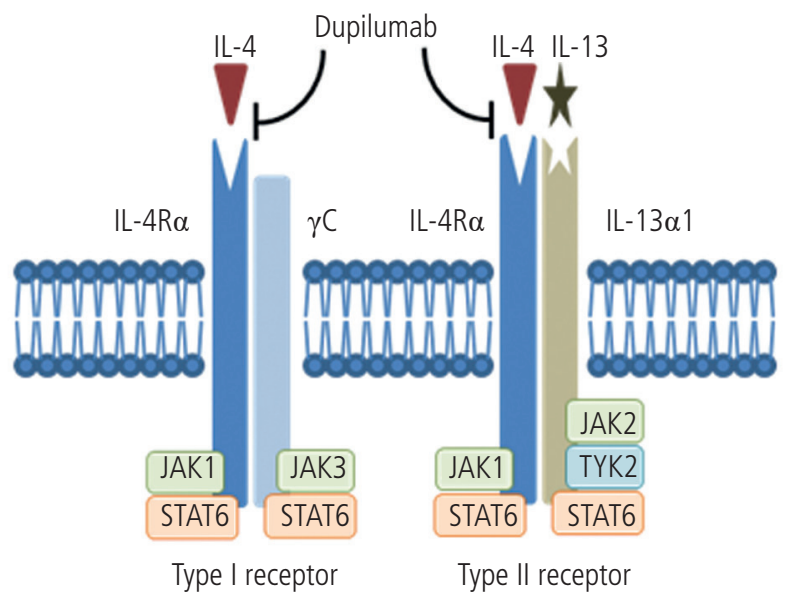

Figure. Mechanism of action of dupilumab. Dupilumab is a fully human monoclonal antibody against IL-4R $\alpha$ that inhibits IL-4/IL-13 signaling and targets type I (IL-4R $\alpha / \gamma \mathrm{C})$ and type II (IL-4R / IL-13R $\alpha 1)$ receptors. Downstream signaling from these receptors, which is mainly mediated by phosphorylated STAT6, activates expression of numerous $T_{H} 2$-related genes, including those associated with IgE class switching.
IL-4 has been shown to stimulate IgE production from B cells, and expression of IL-13 correlates with disease severity and flares [39]. In asthma, IL-4 plays a major role in $\mathrm{T}_{\mathrm{H}} 2$ cell proliferation, cytokine production, and IgE synthesis, and IL-13 has a major role in the pathological features of disease (mucus production, airway hyperresponsiveness, and collagen deposition) [21].

In $\mathrm{AD}$, the cytokines IL-4 and IL-13 modulate the epidermal barrier [41] and inhibit antimicrobial peptide production [42]. IL-13 mRNA levels correlate with disease severity in lesional skin [43], and this cytokine is a potent stimulator of dermal inflammation and remodeling [44,45]. However, much remains to be known about the signaling processes (receptor distribution, utilization, assembly, and affinity for IL-4 and IL-13) and the activated genes that ultimately lead to allergic inflammatory states.

Evidence from basic research and a very large body of evidence from clinical trials supports the model by which dupilumab binding to IL-4 receptors inhibits their activation by IL-4 and IL-13, thus blocking the signaling pathways involved in the development and progression of allergic responses.

\section{Dupilumab: Clinical Trials}

\section{Dupilumab for $A D$}

The first trials on the safety and efficacy of dupilumab for the treatment of moderate-to-severe AD in adults were published in 2013. Large-scale phase II and III trials have been completed and confirm the benefits of the drug for adult AD cases (see below and Table 1); dupilumab was licensed by the FDA and the EMA in 2017 [23,24]. The main parameters for assessment of efficacy in clinical studies are generally the Investigator's Global Assessment (IGA), which measures overall severity of $\mathrm{AD}$ on a 6 -point scale $(0=$ totally clear to $5=$ very severe)[46], and the Eczema Area and Severity Index (EASI), a scale for the assessment of severity and extent of $\mathrm{AD}$ and specifically designed for drug evaluation and clinical trials [47].

\section{Phase I Studies}

Phase I studies (NCT01259323, NCT01385657, NCT01015027) did not reveal safety concerns related to dupilumab (doses of up to $300 \mathrm{mg}$ for 12 weeks) compared with placebo [48]. Inhibition of the $\mathrm{T}_{\mathrm{H}} 2$ immune pathway was demonstrated by significant suppression of thymus and activation regulated chemokine (TARC) and $\mathrm{IgE}$ in patients treated with dupilumab [48]. A pharmacokinetic study of the pooled participants of 6 phase I and II studies $(\mathrm{N}=197)$ revealed no differences in the rate of production of IL-4R $\alpha$ between healthy volunteers and patients with $\mathrm{AD}$ or between the sexes [49].

\section{Phase II Studies}

A phase IIA trial carried out in European centers (NCT01548404) involving adults with moderate-to-severe AD despite treatment with topical corticosteroids and calcineurin inhibitors [50] showed that dupilumab monotherapy for 12 weeks significantly improved the proportion of patients who 


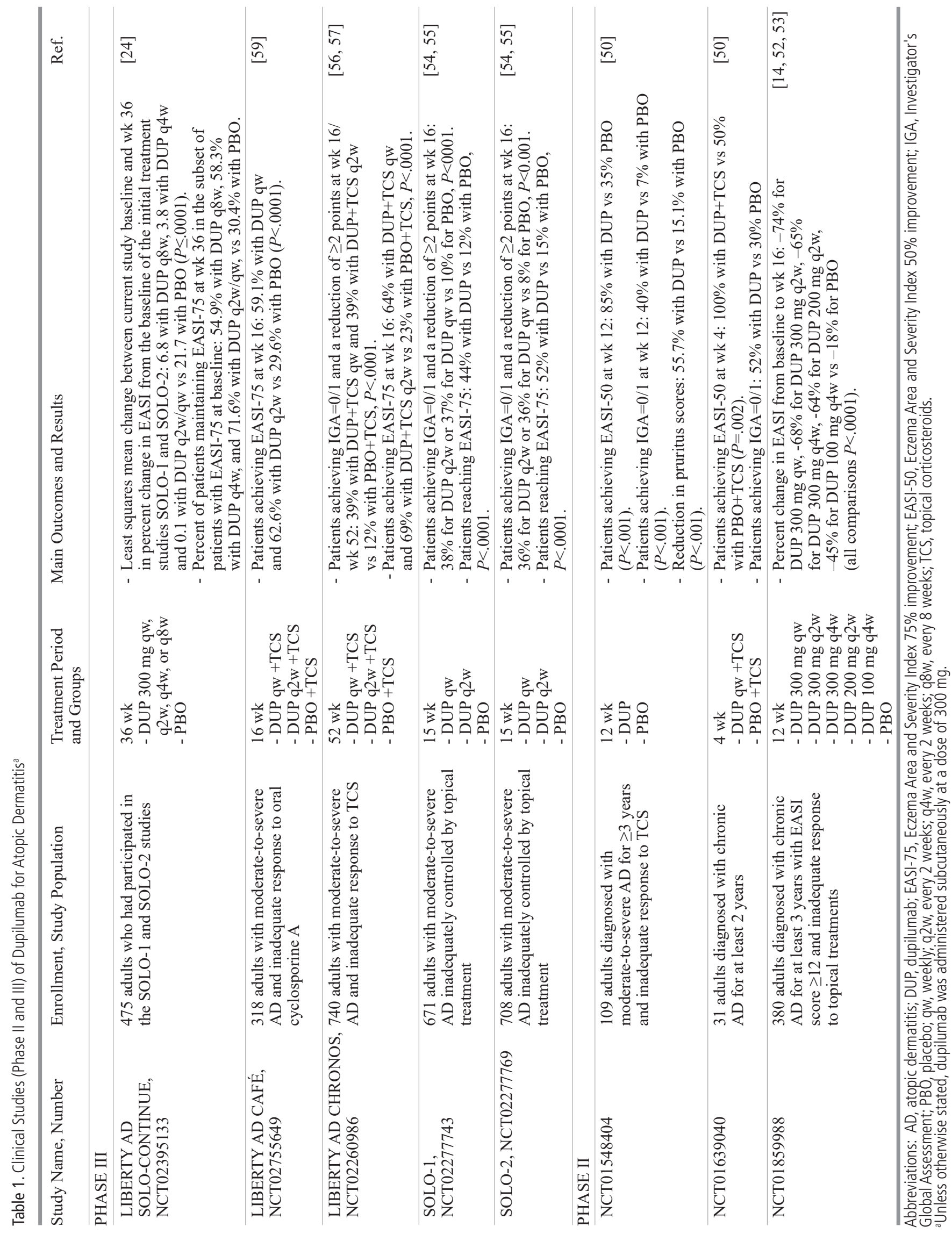


reached EASI-50 ( $85 \%$ of patients in the dupilumab group vs $35 \%$ of those in the placebo group, $P<.001)$ and the IGA requirement $(40 \%$ of patients in the dupilumab group vs $7 \%$ in the placebo group, $P<.001)$ and led to a decrease in pruritus scores $(55.7 \%$ in the dupilumab group vs $15.1 \%$ in the placebo group, $P<.001$ ) [50]. In addition, the trial showed that biomarkers in serum and tissue indicated normalization of $\mathrm{AD}$-affected skin compared with normal skin in patients treated with dupilumab [50] and that the QOL index for AD (QoLIAD) score improved significantly after 12 weeks of dupilumab treatment compared with placebo (mean percent changes of -64.0 [6.91] vs -11.1 [9.31], respectively) [51]. Furthermore, these QoLIAD scores significantly $(P<.05)$ correlated with changes in efficacy outcomes, including EASI $(r=0.4355), 5$-dimensional pruritus $(r=0.4937)$, pruritus numerical rating scale $(\mathrm{r}=0.4064)$, total SCORAD $(\mathrm{r}=0.5559)$, and SCORAD VAS scores for sleep $(\mathrm{r}=0.4681)$ and pruritus $(\mathrm{r}=0.5400)$ [51].

In another phase II trial (NCT01639040) in which dupilumab was administered to 31 adults diagnosed with chronic $\mathrm{AD}$ for at least 2 years in combination with background topical corticosteroids, $100 \%$ of patients in the dupilumab group achieved EASI-50 in 4 weeks, compared with $50 \%$ of the patients in the placebo group $(P=0.002)$ [50].

Finally, Thaci et al [52] performed a large phase IIB trial (NCT01859988) of 380 adult patients from several countries with moderate-to-severe AD inadequately controlled by topical treatments (Table 1). The reductions in the EASI scores were very significantly favorable for all dupilumab treatments compared with placebo $(P<.0001)$, with a reduction of up to $74 \%$ in the case of dupilumab $300 \mathrm{mg}$ once a week compared with $18 \%$ for placebo [52]. In addition, this study made it possible to describe and quantify the large and multidimensional burden of disease associated with AD in the adult population [14]. Of note, treatment with dupilumab improved patient-reported outcomes (PROs) in sleep, mental health, and health-related QOL $[14,52,53]$.

\section{Phase III studies}

SOLO-1 and SOLO-2 (NCT02277743 and NCT02277769, respectively) were large international, prospective, doubleblind, placebo-controlled trials of identical design with the goal of evaluating the efficacy and safety of dupilumab in monotherapy [54]. The participants (671 patients in SOLO-1 and 708 in SOLO-2) were adults with a diagnosis of moderateto-severe $\mathrm{AD}$ inadequately controlled by topical treatment. Prohibited concomitant medications during the trials included topical or systemic corticosteroids and immunomodulating biologic agents. The primary outcome of the studies was the proportion of patients who had both an IGA score of 0 or 1 and a reduction of 2 or more points in that score from baseline at week 16. In SOLO-1/2, this endpoint was met by $38 \% / 36 \%$ of patients taking dupilumab every other week and by $37 \% / 36 \%$ of patients taking dupilumab weekly, compared with $10 \% / 8 \%$ of patients taking placebo $(P<.001$ for all comparisons with placebo) [54]. Similarly, the percentages of patients reaching EASI-75 at week 16 were significantly higher for patients receiving dupilumab than for those receiving placebo (44\%/52\% vs $12 \% / 15 \%, P<.0001)$. Reduction in pruritus and symptoms of anxiety or depression and improvement in QOL and other PROs were also significantly better in patients receiving the drug [54]. The disease burden of $\mathrm{AD}$, as measured by impaired health-related QOL, was reduced significantly in the dupilumab groups relative to the placebo group and was clinically significant [55].

The LIBERTY AD CHRONOS study (NCT02260986) evaluated the long-term (1-year) efficacy and safety of dupilumab in combination with medium-potency topical corticosteroids (TCSs) in adults with moderate-to-severe AD (Table 1) [56-58]. The results at week 16 were maintained at 1 year of treatment, showing a strong improvement in signs and symptoms of $\mathrm{AD}$ in the groups treated with dupilumab plus TCSs. IGA 0/1 was achieved by $39 \%$ of the patients who received dupilumab plus TCSs every week and 39\% of those who received dupilumab every 2 weeks compared with $12 \%$ of those who received placebo $(P<.0001)$. Furthermore, EASI-75 was achieved by $64 \%$ of the patients who received dupilumab plus TCSs every week and $69 \%$ of those who received dupilumab every 2 weeks compared with $23 \%$ who received placebo $(P<.0001)[56,57]$.

The LIBERTY AD CAFÉ (NCT02755649) trial was designed to evaluate dupilumab in combination with TCSs in adults with $\mathrm{AD}$ and an inadequate response or intolerance to cyclosporine A, or for whom cyclosporine A was medically inadvisable (Table 1) [59]. This study included data from 318 patients who were randomized to 2 dupilumab regimens (once- or twice-weekly injections) and placebo, in all cases with concomitant TCSs. The main endpoint, EASI-75, was achieved by $59.1 \%$ and $62.6 \%$ of the patients in the treatment groups with weekly or biweekly dupilumab treatments, respectively, compared with $29.6 \%$ for placebo $(P<.0001)$. Dupilumab in both regimens also significantly improved other clinical outcomes and AD symptoms, including pruritus, pain, sleep disturbance, symptoms of anxiety and depression, and QOL [59].

The LIBERTY AD CONTINUE (NCT02395133) trial was an extension study with 475 adults who had participated in SOLO-1 and SOLO-2. The results showed that patients who continued with the dosing regimen of SOLO 1 and SOLO 2 (300 mg every 2 weeks or every week) experienced an optimal effect in maintaining clinical response, while efficacy for other dose regimens diminished in a dosedependent manner [24].

\section{Ongoing Trials}

A large, open-label, phase III study (LIBERTY AD SOLOMAINTAIN, NCT01949311) is currently investigating the extended efficacy and safety of dupilumab in about 2000 adults who had participated in previous studies. Its results are expected in December 2018. The main endpoints will include the proportion of patients with IGA=0-1, EASI-75, and low disease activity at each visit, as well as any adverse effects detected up to 164 weeks [60].

A study (NCT03389893) to determine the effect of a 6-week course of dupilumab on the host-microbe interface (cutaneous microbial community structure, skin barrier biology, and circulating T-cell profiles) in chronic moderateto-severe AD patients was started in 2018. 


\section{Dupilumab for Asthma}

Dupilumab has been assessed in clinical trials in patients with moderate-to-severe persistent uncontrolled asthma for the last 10 years. Phase I studies (NCT01537653, NCT01537640, NCT01484600, NCT01015027) were concluded in 2012, but data are not yet available. Data from the phase II and III studies are now available and show that dupilumab is efficient in that it can significantly improve lung function (increasing $\mathrm{FEV}_{1}$ ), reduce exacerbations, and reduce the use of maintenance corticosteroids, in this population of patients with limited therapeutic choices (Table 2). The Phase III trial completed in 2017 was the first study with a biologic to show benefits in patients with severe corticosteroid-dependent asthma, regardless of blood eosinophil level or any other type 2 biomarkers at baseline.

\section{Phase II Studies}

Data from the first phase II study (NCT01312961) were published in 2013 (Table 2) [61]. The study was a 12week, randomized, placebo-controlled, parallel-group trial that recruited 104 adult patients with moderate-to-severe eosinophilic asthma not adequately controlled by inhaled corticosteroids (ICSs) and a long-acting $\beta_{2}$ agonist (LABA). Participants had been diagnosed with asthma for at least 12 months and had a high eosinophil count in sputum ( $\geq 3 \%)$ and blood ( $\geq 300$ cells $/ \mu \mathrm{L})$. They were randomly assigned to receive once-weekly subcutaneous injections of dupilumab $300 \mathrm{mg}$ or placebo. During the trial, patients suspended their ICS treatment at week 4 and LABA treatment from weeks 6 to 9 , with the main objective of studying the occurrence of asthma exacerbation. Of the 52 participants in the dupilumab group, only $2(6 \%)$ had an asthma exacerbation, compared with 23 (44\%) who experienced exacerbations in the placebo group (odds ratio, $0.08 ; 95 \% \mathrm{CI}, 0.02-0.28 ; P<.001$ ). In the dupilumab group, $2 \%$ of patients required reliever medication and experienced a reduction in morning peak expiratory flow, compared with $19 \%$ in the placebo group. Forced expiratory volume in 1 second $\left(\mathrm{FEV}_{1}\right)$ improved by more than $200 \mathrm{~mL}$ in patients treated with dupilumab compared with placebo, and this effect continued even after treatment discontinuation. Other parameters that also improved in the dupilumab group included morning asthma symptom score, ACQ-5 score, use of albuterol, and nocturnal awakenings. The biomarkers FeNO, eotaxin-3, serum IgE, and TARC decreased with dupilumab, consistent with the known molecular mechanism of dupilumab.

The second pivotal phase II study (NCT01854047) included a similar but much larger group $(\mathrm{N}=776)$ of patients diagnosed with moderate-to-severe uncontrolled asthma for $>12$ months who had received ICSs at medium-to-high doses and a LABA twice daily (Table 2) [62]. The patients were distributed in 5 groups, receiving $200 \mathrm{mg}$ or $300 \mathrm{mg}$ of dupilumab every 2 or 4 weeks or placebo. The study met the primary endpoint, change in $\mathrm{FEV}_{1}$ from baseline, which increased significantly (except for the dose of $200 \mathrm{mg}$ every 4 weeks) and ranged from 0.35 to $0.43 \mathrm{~L}$ (at 12 weeks) and from 0.17 to $0.26 \mathrm{~L}$ when compared with placebo. Additionally, treatment with dupilumab administered every 2 weeks was associated with a reduced (70\%-70.5\%) risk of the annualized severe exacerbation event rates, significant improvements in ACQ-5 and AQLQ scores at week 24 when compared with placebo, and significant decreases in FeNO. In general, this study proved that dupilumab increased lung function and reduced severe exacerbations in patients with uncontrolled persistent asthma, irrespective of baseline eosinophil counts. A recent post hoc analysis of the results of this study was performed to examine the effect of dupilumab on the 22-item Sino-Nasal Outcome Test (SNOT-22) total score in asthma patients with comorbid perennial allergic rhinitis. It concluded that dupilumab $300 \mathrm{mg}$ every 2 weeks significantly improved allergic rhinitis symptoms in patients with uncontrolled persistent asthma and comorbid perennial allergic rhinitis [63].

\section{Phase III Studies}

The results of two large phase III studies were completed in 2017 , and their results were recently made available in part. The primary endpoints were met in both cases [29,30,32,33].

The pivotal LIBERTY ASTHMA QUEST trial (NCT02414854) enrolled 1902 patients (1795 adults and 107 adolescents) across 413 study sites worldwide. The study analyzed treatment with dupilumab in a broad population of patients with uncontrolled, persistent asthma. All patients continued on a medium or high dose of ICSs and up to 2 additional controller medicines throughout the study. Dupilumab at a dose of $300 \mathrm{mg}$ reduced severe asthma attacks by $46 \%$ in the overall population, and $60 \%$ or $67 \%$ in patients with $\geq 150$ or $\geq 300$ eosinophils $/ \mu \mathrm{L}$, respectively $(P<.001)$. The extent of the patient's response correlated with allergic or atopic status, as reflected by blood eosinophils and other markers. A poorer response was observed in patients with $\leq 150$ eosinophils $/ \mu \mathrm{L}$. The results for the $200 \mathrm{mg}$ and $300 \mathrm{mg}$ dupilumab dose groups were generally comparable for both exacerbations and $\mathrm{FEV}_{1}$ (Table 2). The results of prebronchodilator $\mathrm{FEV}_{1}$ at 12 weeks were equally encouraging, as the mean improvement with dupilumab was $130 \mathrm{~mL}(9 \%)$ for the general population and $210 \mathrm{~mL}(11 \%)$ or $240 \mathrm{~mL}(18 \%)$ in patients with $\geq 150$ or $\geq 300$ eosinophils $/ \mu \mathrm{L}$, respectively $(P<.001)$.

The LIBERTY ASTHMA VENTURE study (NCT02528214) enrolled 210 patients (103 in the dupilumab arm and 107 in the placebo arm) with severe asthma and regular use of maintenance oral corticosteroids (OCSs, prednisone or prednisolone) in the 6 months prior to enrolment (Table 2)[31]. The study showed that dupilumab reduced the use of maintenance OCSs by $70 \%$ (median reduction of $100 \%$ ) compared with $42 \%$ in the case of placebo (median reduction of $50 \%)(P<.0001)$. In analyses of patients with baseline eosinophil counts $\geq 300$ cells $/ \mu \mathrm{L}$, the decrease was even greater, with OCS use reduced by $80 \%$ on average with dupilumab compared with $43 \%$ for placebo $(P=.0001)$. At 24 weeks, despite the reduced use of OCSs, patients treated with dupilumab had 59\% fewer attacks (exacerbations) in the overall population $(P<.0001)$ and $71 \%$ fewer attacks in patients with eosinophil counts $\geq 300$ cells $/ \mu \mathrm{L}$. Also at 24 weeks, dupilumab improved lung function compared with placebo by $220 \mathrm{~mL}$ $(15 \%)$ in the overall population, as assessed based on $\mathrm{FEV}_{1}$ $(P=.0007)$ and by $320 \mathrm{~mL}(25 \%)$ in patients with eosinophil counts $\geq 300$ cells $/ \mu \mathrm{L}(P=.0049)$. In the overall population, $80 \%$ of patients who received dupilumab reduced their OCS dose by 


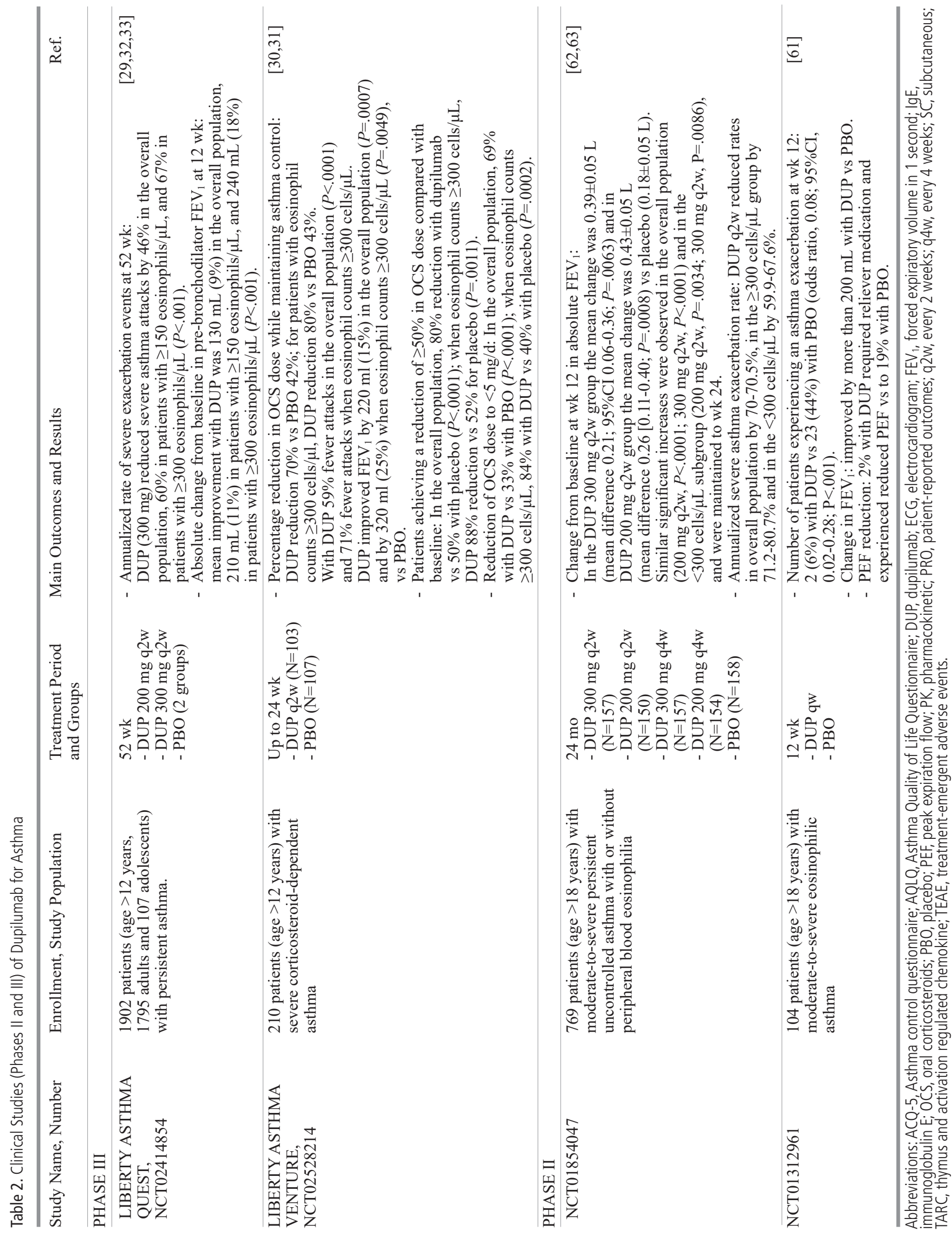


at least half while maintaining overall asthma control compared with $50 \%$ for patients who received placebo $(P<.0001)$. In addition, $69 \%$ of patients who received dupilumab reduced their OCS dose to less than $5 \mathrm{mg}$ per day while maintaining asthma control compared with $33 \%$ of patients who received placebo $(P<.0001)$. This was the first study with a biologic to show a benefit in the population with severe corticosteroiddependent asthma, regardless of blood eosinophil levels or any other type 2 biomarkers at baseline [31].

\section{Ongoing Studies}

Three ongoing studies are analyzing the safety and efficacy of dupilumab in the treatment of moderate-to-severe asthma. LIBERTY ASTHMA EXPEDITION (NCT02573233) is a placebo-controlled study designed to evaluate the effect of dupilumab on airway inflammation in patients with persistent asthma. Its main outcomes are the change from baseline in inflammatory cell count per square millimeter in the bronchial submucosa and change from baseline in the mucin-stained area of the bronchial submucosa per square millimeter, over a period of treatment of 12 weeks. Its results are expected in 2018 [64].

LIBERTY ASTHMA TRAVERSE (NCT02134028) is an open-label extension trial with an enrolment of 2206 adolescent and adult patients who completed the treatment period in a previous dupilumab asthma clinical study. Dupilumab is being administered every 2 weeks for a maximum of 60 or 108 weeks depending on the enrollment date, and the primary endpoint is the evaluation of any adverse events. The study is expected to be completed in October 2019 [65].

The third ongoing study, VOYAGE (NCT02948959), is specifically evaluating dupilumab every 2 weeks (vs placebo) in children aged 6 to $<12$ years with a diagnosis of persistent asthma for $\geq 12$ months. The treatment period will be 52 weeks, and the main endpoint will be the annualized rate of severe exacerbation events during this treatment period. The results are expected by January 2021 [66].

\section{Dupilumab for Chronic Rhinosinusitis With Nasal Polyposis}

Chronic rhinosinusitis with nasal polyposis is characterized by type 2 inflammation with elevated levels of IL-5, IL-13, and eosinophils in the polyps [3]. Driven by the success of dupilumab in the treatment of other $\mathrm{T}_{\mathrm{H}} 2$-mediated diseases, a phase II study (NCT01920893) was carried out to evaluate the efficacy of dupilumab in the treatment of bilateral nasal polyposis and chronic symptoms of sinusitis [67]. This randomized, double-blind, placebo-controlled study assessed the endoscopic nasal polyp score (NPS) and symptoms of sinusitis after a 16-week treatment with dupilumab. The population analyzed consisted of 60 adults with chronic sinusitis and nasal polyposis refractory to intranasal corticosteroids. The results, published in 2016, revealed that dupilumab added to mometasone furoate nasal spray reduced the endoscopic nasal polyp burden after 16 weeks when compared with mometasone alone [68]. In addition, significant improvements with dupilumab were observed for the 22-item
SinoNasal Outcome Test and for the sense of smell, assessed using the University of Pennsylvania Smell Identification Test. Furthermore, a longitudinal analysis of plasma CCL26, an eosinophil chemoattractant that is characteristically increased in rhinosinusitis with nasal polyposis, showed a decrease in levels after treatment with dupilumab.

Two phase III clinical studies, both due to be completed in 2018, evaluate the efficacy of dupilumab in reducing the severity of nasal congestion/obstruction and endoscopic NPS in adult patients also treated with intranasal corticosteroids for 24 weeks (SINUS-24, NCT02912468, estimated N=240) or a 52-week treatment period (SINUS-52, NCT02898454, estimated $\mathrm{N}=360)[69,70]$.

\section{Dupilumab for Eosinophilic Esophagitis}

EoE has only been considered a chronic allergic/immune condition in recent decades [71]. Patients with EoE present inflammation of the esophagus, with abundant eosinophilia. Although many aspects of the pathophysiology of this disease remain unclear, the excess of eosinophils in the esophageal epithelium points to the recruiting action of $\mathrm{T}_{\mathrm{H}} 2$ cytokines IL-4, IL-5, and IL-13 [71,72]. For this reason, dupilumab was considered a possible therapy for this disease.

A phase II, randomized, double-blind, placebo-controlled clinical trial (NCT02379052) was carried out with 47 participants to assess the clinical efficacy of dupilumab for relieving symptoms in adult patients with active, moderateto-severe EoE [73]. The study was completed in July 2017 and the preliminary results were presented at the World Congress of Gastroenterology the same year [38]. The primary endpoint of the study was the change from baseline to week 10 in the Straumann Dysphagia Instrument (SDI) score, a patient-reported measure of swallowing difficulty on a 0-9 point scale, with 9 indicating more severe symptoms. A total of 47 patients were randomized to 2 treatment groups in this 12-week treatment study, and both groups had a mean baseline SDI score of 6.4. Patients received either dupilumab $300 \mathrm{mg}$ weekly following a 600 -mg loading dose or placebo. At week 10 , patients who received dupilumab reported a significant improvement in the ability to swallow with a 3-point reduction in their SDI score (45\% improvement) compared with 1.3 points ( $19 \%$ improvement) for those patients who received placebo $(P=.0304)$ [38]. Notably, the mean percent change in overall peak intraepithelial eosinophil count from baseline to 12 weeks was significantly reduced by $93 \%$ from baseline in patients who received dupilumab weekly compared with an increase of $14 \%$ in those who received placebo $(P<.0001)$ [38], thus highlighting the action of dupilumab against the root mechanism of the disease.

In conclusion, the study revealed that dupilumab significantly improved dysphagia, esophageal eosinophil counts, endoscopic features, histology, and esophageal distensibility in adults with active EoE compared with placebo [38].

Dupilumab recently received orphan drug status for the treatment of EoE from the Orphan Drug Designation program of the FDA [38]. 


\section{Safety and Tolerability of Dupilumab}

In all $\mathrm{AD}$ trials, the incidence of adverse events was similar in the dupilumab groups and the placebo groups. Serious adverse events and adverse events leading to treatment discontinuation were uncommon $[14,54,56,59,74]$. However, in long-term studies, the rates of conjunctivitis, injection site reactions, and local herpes simplex infections were higher in the dupilumab groups than in the placebo groups [56,57]. Two recent meta-analyses of randomized clinical trials of dupilumab for the treatment of AD showed that treated patients presented a lower risk of skin infection (risk ratio, 0.54; 95\%CI, 0.42-0.69) [75] and eczema herpeticum [76]. Furthermore, the risk decreased for exacerbation of AD (risk ratio, 0.44; 95\%CI, 0.34-0.59) [75]. However, the risks were higher in the groups treated with dupilumab for injection site reactions (risk ratio, 2.24; 95\% CI, 1.68-2.99), headache (risk ratio, 1.47; 95\% $\mathrm{CI}, 1.05-2.06$ ), and conjunctivitis (risk ratio, $2.64 ; 95 \% \mathrm{CI}, 1.79-3.89)$ than in patients treated with placebo [75]. Nasopharyngitis, urinary tract infection, upper respiratory tract infection, and herpes virus infection were equally distributed between the dupilumab groups and placebo groups [75].

In the SOLO-1 and SOLO-2 studies, as well as in the LIBERTY AD CHRONOS study, the longest-running study (1 year) for which there are available data, the rates of conjunctivitis (allergic or unspecified) were higher in the dupilumab groups than in the placebo groups $[54,56,57]$. It has been speculated that this increment in the frequency of conjunctivitis could limit the use of dupilumab in the treatment of allergic diseases, especially in children [77]. However, in the SOLO trials, more than $90 \%$ of the adverse events of conjunctivitis were mild or moderate in severity, and more than $75 \%$ resolved or were resolving during study treatment (only 1 of 920 patients treated with dupilumab had to discontinue because of conjunctivitis). Importantly, other dupilumab trials involving patients with asthma or nasal polyposis did not show a higher rate of conjunctivitis than placebo, suggesting an AD-specific mechanism [74]. Furthermore, the incidence of atopic keratoconjunctivitis was less than $0.5 \%$ across all groups and was no higher in the dupilumab group than in the placebo group [74]. The causes of the increased incidence of conjunctivitis in trials investigating dupilumab for the treatment of $\mathrm{AD}$ remain unknown, and further research will be required to evaluate the overall effect of the drug in AD therapy. Although the effect of IL-13 on regulation of conjunctival goblet cell density is not completely understood, the fact that IL-13-deficient mice have a significantly lower number of filled conjunctival goblet cells than wild-type mice suggested a potential role of IL-13 in regulating conjunctival goblet cells and, therefore, the quality of tears. This could compromise the protective function of tears, thereby contributing to ocular surface damage and inducing dry eye syndrome in some patients. In fact, the use of artificial tears is recommended in patients receiving dupilumab [78,79]. The presence of inflammation of the anterior conjunctiva and hyperemia of the limbus was recently reported in $25 \%-50 \%$ of AD patients treated with dupilumab. The condition was successfully treated with fluorometholone $0.1 \%$ eye drops or tacrolimus $0.03 \%$ eye ointment [80].
In the LIBERTY ASTHMA QUEST study, a long-term analysis, the overall rates of adverse events, deaths, infections, conjunctivitis, herpes, and discontinuations were comparable between the dupilumab and the placebo groups. Injection site reactions were more common in the dupilumab groups $(17 \%$ vs $8 \%$ ) [29,32,33]. Transient increases in serum eosinophil levels have been reported in some asthmatic patients at the beginning of treatment with dupilumab.

In all the dupilumab clinical trials, including those with treatment periods of 52 weeks, only $10 \%$ of patients developed antidrug antibodies. In most cases, the antibody titer was low and the effect transitory ( $<1 \%$ persistent) [24]. Given the low incidence of immunogenicity, its relevance for efficacy or safety could not be determined. The LIBERTY AD SOLOMAINTAIN clinical trial (NCT01949311), which comprises 164 weeks of treatment, will analyze the long-term safety and immunogenicity of dupilumab [60].

Dose adjustment is not recommended in elderly patients ( $\geq 65$ years) or as a function of body weight and is not needed in patients with mild or moderate kidney impairment. The efficacy and safety profile of dupilumab has not been established in patients with liver impairment or in patients aged $\leq 18$ years $[23]$.

Taken together, the clinical data suggest that dupilumab is generally well tolerated and that the common adverse events are mild and manageable. Long-term data on AD and asthma reported from clinical trials support the view that dupilumab is very safe, although data from extension studies and in children (both for AD and asthma) will definitely clarify this issue.

\section{Dupilumab: Implications for Clinical Practice}

Dupilumab is the first biologic that effectively addresses the pathophysiology of allergic syndromes, the $\mathrm{T}_{\mathrm{H}} 2$-driven inflammatory pathway. The FDA approval of dupilumab has paved the way for biologic therapy to be used as treatment of moderate-to-severe AD. Compared with other biologics (eg, nemolizumab, lebrikizumab, tralokinumab), dupilumab is currently the only biologic with robust evidence of efficacy in $\mathrm{AD}$ [26].

Treatment with dupilumab is also potentially useful in patients with moderate-to-severe asthma who are not adequately controlled with ICS+LABA [30,81], since it enables reduction or suspension of systemic corticosteroids, decreases exacerbations, and improves lung function. Furthermore, dupilumab has proven to be effective in asthmatic patients independent of eosinophil levels in blood [62], while the biologics that block the IL-5/IL-5R $\alpha$ axis (mepolizumab, reslizumab, and benralizumab) are restricted to uncontrolled severe asthma patients with $>300$ eosinophils/ $\mu \mathrm{L}$ (mepolizumab, benralizumab) or $>400$ eosinophils $/ \mu \mathrm{L}$ (reslizumab) [82].

Another major advantage of dupilumab could be that it effectively addresses AD/asthma and their main comorbidities (eg, chronic sinusitis, nasal polyposis). Dupilumab could therefore have a major therapeutic impact on several allergic diseases simultaneously. 
In a decade of intense clinical research, dupilumab has proven an efficacious and safe tool in the management of AD and asthma in its more severe presentations and for which there were no therapeutic alternatives. As data from new studies become available, it is likely that dupilumab will show its potential in a wider range of allergic diseases and lead the way to the targeted modulation of the immune system for therapeutic purposes.

\section{Acknowledgments}

We wish to thank Francisco de López de Saro (Trialance SCCL) for medical writing support.

\section{Funding}

The authors declare that no funding was received for the present study.

\section{Conflicts of Interest}

JS reports having served as a consultant for Thermo Fisher, Novartis, Sanofi, Leti, FAES FARMA, Mundipharma, and GSK. He has also received lecture fees from Novartis, GSK, Stallergenes, LETI, and FAES FARMA and grant support for research from Thermo Fisher and ALK.

ID has participated in advisory boards for ALK, AstraZeneca, FAES FARMA, GSK, Novartis, Sanofi, and Stallergenes. He has also received lecture fees from ALK, Astra-Zeneca, FAES FARMA, GSK, Leti, Menarini, Novartis, Sanofi, Stallergenes, and Teva and grant support for research from Merck and Thermo Fisher.

\section{References}

1. Wollenberg A, Oranje A, Deleuran M, Simon D, Szalai Z, Kunz B, et al. ETFAD/EADV Eczema task force 2015 position paper on diagnosis and treatment of atopic dermatitis in adult and paediatric patients. J Eur Acad Dermatol Venereol. 2016;30:729-47.

2. Global Initiative for Asthma. Global strategy for asthma management and prevention. 2017 [December 22, 2017]; Available from: www.ginasthma.org.

3. Stevens WW, Schleimer RP, Kern RC. Chronic Rhinosinusitis with Nasal Polyps. J Allergy Clin Immunol Pract. 2016:4:565 72.

4. Pascual M, Davila I, Isidoro-Garcia M, Lorente F. Epigenetic aspects of the allergic diseases. Front Biosci (Schol Ed). 2010;2:815-24.

5. Mallol J, Crane J, von Mutius E, Odhiambo J, Keil U, Stewart A. The International Study of Asthma and Allergies in Childhood (ISAAC) Phase Three: a global synthesis. Allergol Immunopathol (Madr). 2013;41:73-85.

6. Ellis CN, Mancini AJ, Paller AS, Simpson EL, Eichenfield LF. Understanding and managing atopic dermatitis in adult patients. Semin Cutan Med Surg. 2012;31:S18-22.

7. Silvestre Salvador JF, Romero-Perez D, Encabo-Duran B. Atopic Dermatitis in Adults: A Diagnostic Challenge. J Investig Allergol Clin Immunol. 2017;27:78-88.
8. Sicras-Mainar A, Navarro-Artieda R, Sánchez L, Sastre J. Prevalence of severe atopic dermatitis in adults in 3 areas of Spain. J Invest Allergol Clin Immunol. 2018;in press.

9. World Allergy Organization. Rhinitis. [February 7, 2018] Available from: http://www.worldallergy.org/professional/ allergic_diseases_center/rhinitis/rhinitissynopsis.php.

10. Hastan D, Fokkens WJ, Bachert C, Newson RB, Bislimovska J, Bockelbrink $A$, et al. Chronic rhinosinusitis in Europe-an underestimated disease. A GA2LEN study. Allergy. 2011;66:1216-23.

11. Dellon ES, Hirano I. Epidemiology and Natural History of Eosinophilic Esophagitis. Gastroenterology. 2017

12. Koplin JJ, Mills EN, Allen KJ. Epidemiology of food allergy and food-induced anaphylaxis: is there really a Western world epidemic? Curr Opin Allergy Clin Immunol. 2015;15:409-16.

13. Tang ML, Mullins RJ. Food allergy: is prevalence increasing? Intern Med J. 2017:47:256-61.

14. Simpson EL, Bieber T, Eckert L, Wu R, Ardeleanu M, Graham $N M$, et al. Patient burden of moderate to severe atopic dermatitis (AD): Insights from a phase $2 b$ clinical trial of dupilumab in adults. J Am Acad Dermatol. 2016;74:491-8.

15. Saunes M, Oien T, Dotterud CK, Romundstad PR, Storro $O$ Holmen $\mathrm{TL}$, et al. Early eczema and the risk of childhood asthma: a prospective, population-based study. BMC Pediatr. 2012;12:168.

16. Drucker AM, Wang AR, Li WQ, Sevetson E, Block JK, Qureshi AA. The Burden of Atopic Dermatitis: Summary of a Report for the National Eczema Association. J Invest Dermatol. 2017;137:26-30.

17. Porsbjerg C, Menzies-Gow A. Co-morbidities in severe asthma: Clinical impact and management. Respirology. 2017;22:65161.

18. Woodruff PG, Modrek B, Choy DF, Jia G, Abbas AR, Ellwanger $A$, et al. T-helper type 2-driven inflammation defines major subphenotypes of asthma. Am J Respir Crit Care Med. 2009; 180:388-95

19. Kim DH, Park KY, Kim BJ, Kim MN, Mun SK.Anti-immunoglobulin $E$ in the treatment of refractory atopic dermatitis. Clin Exp Dermatol. 2013:38:496-500.

20. Doran E, Cai F, Holweg CTJ, Wong K, Brumm J, Arron JR Interleukin-13 in Asthma and Other Eosinophilic Disorders. Front Med (Lausanne). 2017;4:139.

21. Gour N, Wills-Karp M. IL-4 and IL-13 signaling in allergic airway disease. Cytokine. 2015;75:68-78.

22. Mullard A. FDA approves dupilumab for severe eczema. Nat Rev Drug Discov. 2017;16:305.

23. US Food and Drug Administration. Dupixent Prescribing Information (approved March 28, 2017). [December 21, 2017]; Available from: https://www.accessdata.fda.gov/ drugsatfda_docs/label/2017/761055lbl.pdf.

24. European Medicines Agency. Dupixent Summary of Product Characteristics (approved October 11, 2017). [December 21, 2017]; Available from: http://www.ema.europa.eu/docs/ en_GB/document_library/EPAR_-_Product_Information/ human/004390/WC500236507.pdf.

25. Roekevisch E, Spuls PI, Kuester D, Limpens J, Schmitt J. Efficacy and safety of systemic treatments for moderate-to-severe atopic dermatitis: a systematic review. J Allergy Clin Immunol. 2014;133:429-38 
26. Snast I, Reiter O, Hodak E, Friedland R, Mimouni D, Leshem YA. Are Biologics Efficacious in Atopic Dermatitis? A Systematic Review and Meta-Analysis. Am J Clin Dermatol. 2017.

27. Sastre J, Fabbri LM, Price D, Wahn HU, Bousquet J, Fish JE, et al. Insights, attitudes, and perceptions about asthma and its treatment: a multinational survey of patients from Europe and Canada. World Allergy Organ J. 2016;9:13.

28. Barranco P, Phillips-Angles E, Dominguez-Ortega J, Quirce S. Dupilumab in the management of moderate-to-severe asthma: the data so far. Ther Clin Risk Manag. 2017;13:1139-49.

29. Sanofi-Regeneron. Sanofi and Regeneron Announce Positive Dupilumab Topline Results From Phase 3 Trial in Uncontrolled Persistent Asthma. Press Release, September 11, 2017

30. Sanofi-Regeneron. Dupilumab Significantly Reduced Steroid Use, Asthma Attacks, and Improved Lung Function in a Phase 3 Study of People with Severe Steroid-Dependent Asthma. Press Release, October 31, 2017.

31. Rabe KF, Nair P, Brusselle G, Maspero JF, Castro M, Sher L, et al. Efficacy and Safety of Dupilumab in GlucocorticoidDependent Severe Asthma. N Engl J Med. 2018.

32. Busse WW, Maspero JF, Rabe KF, Papi A, Wenzel SE, Ford LB, et al. Liberty Asthma QUEST: Phase 3 Randomized, DoubleBlind, Placebo-Controlled, Parallel-Group Study to Evaluate Dupilumab Efficacy/Safety in Patients with Uncontrolled, Moderate-to-Severe Asthma. Adv Ther. 2018.

33. Castro M, Corren J, Pavord ID, Maspero J, Wenzel S, Rabe KF, et al. Dupilumab Efficacy and Safety in Moderate-to-Severe Uncontrolled Asthma. N Engl J Med. 2018.

34. Akdis CA, Bachert C, Cingi C, Dykewicz MS, Hellings PW, Naclerio RM, et al. Endotypes and phenotypes of chronic rhinosinusitis: a PRACTALL document of the European Academy of Allergy and Clinical Immunology and the American Academy of Allergy, Asthma \& Immunology. J Allergy Clin Immunol. 2013;131:1479-90.

35. Peters AT, Spector S, Hsu J, Hamilos DL, Baroody FM, Chandra RK, et al. Diagnosis and management of rhinosinusitis: a practice parameter update. Ann Allergy Asthma Immunol. 2014; 113:347-85.

36. Liacouras CA, Furuta GT, Hirano I, Atkins D, Attwood SE, Bonis PA, et al. Eosinophilic esophagitis: updated consensus recommendations for children and adults. J Allergy Clin Immunol. 2011;128:3-20 e6; quiz 1-2.

37. Lucendo AJ, Molina-Infante J, Arias A, von Arnim U, Bredenoord AJ, Bussmann C, et al. Guidelines on eosinophilic esophagitis: evidence-based statements and recommendations for diagnosis and management in children and adults. United European Gastroenterol J. 2017;5:335-58.

38. Sanofi-Regeneron. Sanofi and Regeneron Announce Positive Phase 2 Study Results for Dupilumab in Patients With Active Moderate-to-Severe Eosinophilic Esophagitis. Press Release, October 16, 2017.

39. May RD, Fung M. Strategies targeting the IL-4/IL-13 axes in disease. Cytokine. 2015;75:89-116.

40. Bao K, Reinhardt RL. The differential expression of IL-4 and IL-13 and its impact on type-2 immunity. Cytokine. 2015;75:25-37.

41. Howell MD, Kim BE, Gao P, Grant AV, Boguniewicz $M$, DeBenedetto $A$, et al. Cytokine modulation of atopic dermatitis filaggrin skin expression. J Allergy Clin Immunol. 2009;124:R7-R12.
42. Ong PY, Ohtake T, Brandt C, Strickland I, Boguniewicz M, Ganz $T$, et al. Endogenous antimicrobial peptides and skin infections in atopic dermatitis. N Engl J Med. 2002;347:1151-60.

43. Nomura I, Goleva E, Howell MD, Hamid QA, Ong PY, Hall CF, et al. Cytokine milieu of atopic dermatitis, as compared to psoriasis, skin prevents induction of innate immune response genes. J Immunol. 2003;171:3262-9.

44. Zheng $\mathrm{T}$, Oh MH, Oh SY, Schroeder JT, Glick AB, Zhu Z. Transgenic expression of interleukin-13 in the skin induces a pruritic dermatitis and skin remodeling. J Invest Dermatol. 2009; 129:742-51.

45. Hussein YM, Ahmad AS, Ibrahem MM, Elsherbeny HM, Shalaby SM, El-Shal AS, et al. Interleukin 13 Receptors as Biochemical Markers in Atopic Patients. J Invest Allergol Clin Immunol. 2011;21:101-7.

46. Barbier N, Paul C, Luger T, Allen R, De Prost Y, Papp K, et al. Validation of the Eczema Area and Severity Index for atopic dermatitis in a cohort of 1550 patients from the pimecrolimus cream $1 \%$ randomized controlled clinical trials programme. $\mathrm{Br}$ J Dermatol. 2004; 150:96-102.

47. Hanifin JM, Thurston M, Omoto M, Cherill R, Tofte SJ, Graeber $M$. The eczema area and severity index (EASI): assessment of reliability in atopic dermatitis. EASI Evaluator Group. Exp Dermatol. 2001;10:11-8.

48. Radin A, Ren H, Papino-Wood P, Chaudhry U, Hamilton JD. First-in-human study of REGN668/SAR231893 (IL-4Ra mAb): Safety, tolerability and biomarker results of a randomized, double-blind, placebo-controlled, single ascending dose study in healthy volunteers. J Allergy Clin Immunol. 2013;131:AB158.

49. Kovalenko P, DiCioccio AT, Davis JD, Li M, Ardeleanu M, Graham N, et al. Exploratory Population PK Analysis of Dupilumab, a Fully Human Monoclonal Antibody Against IL4Ralpha, in Atopic Dermatitis Patients and Normal Volunteers. CPT Pharmacometrics Syst Pharmacol. 2016;5:617-24.

50. Beck LA, Thaci D, Hamilton JD, Graham NM, Bieber T, Rocklin $\mathrm{R}$, et al. Dupilumab treatment in adults with moderate-tosevere atopic dermatitis. N Engl J Med. 2014;371:130-9.

51. Tsianakas A, Luger TA, Radin A. Dupilumab treatment improves quality of life in adult patients with moderate-tosevere atopic dermatitis: Results from a randomized, placebocontrolled clinical trial. Br J Dermatol. 2017.

52. Thaci D, Simpson EL, Beck LA, Bieber T, Blauvelt A, Papp K, et al. Efficacy and safety of dupilumab in adults with moderateto-severe atopic dermatitis inadequately controlled by topical treatments: a randomised, placebo-controlled, dose-ranging phase 2b trial. Lancet. 2016:387:40-52.

53. Simpson EL, Gadkari A, Worm M, Soong W, Blauvelt A, Eckert $L$, et al. Dupilumab therapy provides clinically meaningful improvement in patient-reported outcomes (PROs): A phase $\mathrm{Ilb}$, randomized, placebo-controlled, clinical trial in adult patients with moderate to severe atopic dermatitis (AD). J Am Acad Dermatol. 2016;75:506-15.

54. Simpson EL, Bieber T, Guttman-Yassky E, Beck LA, Blauvelt A, Cork MJ, et al. Two Phase 3 Trials of Dupilumab versus Placebo in Atopic Dermatitis. N Engl J Med. 2016;375:2335-48.

55. Simpson EL. Dupilumab Improves General Health-Related Quality-of-Life in Patients with Moderate-to-Severe Atopic Dermatitis: Pooled Results from Two Randomized, Controlled Phase 3 Clinical Trials. Dermatol Ther (Heidelb). 2017;7:243-8. 
56. Blauvelt $A$, de Bruin-Weller $M$, Gooderham $M$, Cather JC, Weisman J, Pariser $D$, et al. Long-term management of moderate-to-severe atopic dermatitis with dupilumab and concomitant topical corticosteroids (LIBERTY AD CHRONOS): a 1-year, randomised, double-blinded, placebo-controlled, phase 3 trial. Lancet. 2017;389:2287-303.

57. Thomson J, Wernham AGH, Williams HC. Long-term management of moderate-to-severe atopic dermatitis with dupilumab and concomitant topical corticosteroids (LIBERTY AD CHRONOS): a critical appraisal. Br J Dermatol. 2018.

58. Strowd LC, Feldman SR. Dupilumab for atopic dermatitis. Lancet. 2017:389:2265-6.

59. de Bruin-Weller M, Thaci D, Smith $\mathrm{CH}$, Reich $\mathrm{K}$, Cork M, Radin $A$, et al. Dupilumab with concomitant topical corticosteroids in adult patients with atopic dermatitis who are not adequately controlled with or are intolerant to ciclosporin $A$, or when this treatment is medically inadvisable: a placebo-controlled, randomized phase 3 clinical trial (LIBERTY AD CAFE). Br J Dermatol. 2017

60. Sanofi. Open-label Study of Dupilumab (REGN668/ SAR231893) in Patients With Atopic Dermatitis. ClinicalTrials. gov; [January 12, 2018]; Available from: https://clinicaltrials. gov/ct2/show/NCT01949311?term =NCT01949311\&rank=1.

61. Wenzel S, Ford L, Pearlman D, Spector S, Sher L, Skobieranda F, et al. Dupilumab in persistent asthma with elevated eosinophil levels. N Engl J Med. 2013:368:2455-66.

62. Wenzel S, Castro M, Corren J, Maspero J, Wang L, Zhang B, et al. Dupilumab efficacy and safety in adults with uncontrolled persistent asthma despite use of medium-to-high-dose inhaled corticosteroids plus a long-acting beta2 agonist: a randomised double-blind placebo-controlled pivotal phase $2 \mathrm{~b}$ dose-ranging trial. Lancet. 2016;388:31-44.

63. Weinstein SF, Katial R, Jayawardena S, Pirozzi G, Staudinger $H$, Eckert $L$, et al. Efficacy and safety of dupilumab in perennial allergic rhinitis and comorbid asthma. J Allergy Clin Immunol. 2018; in press.

64. Sanofi. Evaluation of Dupilumab's Effects on Airway Inflammation in Patients With Asthma (EXPEDITION). ClinicalTrials.gov; [February 23, 2018]; Available from: https:// clinicaltrials.gov/ct2/show/NCT02573233.

65. Sanofi. Long-Term Safety Evaluation of Dupilumab in Patients With Asthma (LIBERTY ASTHMA TRAVERSE). ClinicalTrials.gov; [January 12, 2018]; Available from: https://clinicaltrials.gov/ ct2/show/NCT02134028?term=NCT02134028\&rank $=1$.

66. Sanofi. Evaluation of Dupilumab in Children With Uncontrolled Asthma (VOYAGE). ClinicalTrials.gov; [January 12, 2018]; Available from: https://clinicaltrials.gov/ct2/show/NCT02948 959?term $=$ NCT02948959\&rank=1.

67. Sanofi. An Evaluation of Dupilumab in Patients With Nasal Polyposis And Chronic Symptoms Of Sinusitis (NCT01920893). [December 28, 2017]; Available from: https://clinicaltrials.gov/ ct2/show/NCT01920893.

68. Bachert C, Mannent L, Naclerio RM, Mullol J, Ferguson BJ, Gevaert $P$, et al. Effect of Subcutaneous Dupilumab on Nasal Polyp Burden in Patients With Chronic Sinusitis and Nasal Polyposis: A Randomized Clinical Trial. JAMA. 2016;315:46979.

69. Sanofi. Controlled Clinical Study of Dupilumab in Patients With Nasal Polyps (SINUS-52). ClinicalTrials.gov; [updated
December 3, 2017]; Available from: https://clinicaltrials.gov/ ct2/show/NCT02898454.

70. Sanofi. A Controlled Clinical Study of Dupilumab in Patients With Nasal Polyps (SINUS-24). ClinicalTrials.gov; [December 3, 2017]; Available from: https://clinicaltrials.gov/ct2/show/ NCT02912468?term $=$ NCT02912468\&rank $=1$.

71. Goyal A, Cheng E. Recent discoveries and emerging therapeutics in eosinophilic esophagitis. World J Gastrointest Pharmacol Ther. 2016;7:21-32.

72. Durrani S, Rothenberg M. Recent advances in eosinophilic esophagitis. F1000Res. 2017;6:1775.

73. Sanofi. Study of Dupilumab in Adult Patients With Active Eosinophilic Esophagitis (EoE), Identifier: NCT02379052. ClinicalTrials.gov; [December 3, 2017];Available from: https:// clinicaltrials.gov/.

74. Simpson EL, Akinlade B, Ardeleanu M. Two Phase 3 Trials of Dupilumab versus Placebo in Atopic Dermatitis. N Engl J Med. 2017;376:1090-1.

75. Ou Z, Chen C, Chen A, Yang Y, Zhou W. Adverse events of Dupilumab in adults with moderate-to-severe atopic dermatitis: A meta-analysis. Int Immunopharmacol. 2018;54:303-10.

76. Fleming $P$, Drucker AM. Risk of infection in patients with atopic dermatitis treated with dupilumab: A meta-analysis of randomized controlled trials. J Am Acad Dermatol. 2018;78:62-9 e1.

77. Mennini M, Dahdah L, Fiocchi A. Two Phase 3 Trials of Dupilumab versus Placebo in Atopic Dermatitis. N Engl J Med. 2017:376:1090.

78. De Paiva CS, Raince JK, McClellan AJ, Shanmugam KP, Pangelinan SB, Volpe EA, et al. Homeostatic control of conjunctival mucosal goblet cells by NKT-derived IL-13. Mucosal Immunol. 2011;4:397-408.

79. Contreras-Ruiz L, Ghosh-Mitra A, Shatos MA, Dartt DA, Masli S Modulation of conjunctival goblet cell function by inflammatory cytokines. Mediators Inflamm. 2013;2013:636812.

80. Wollenberg A, Ariens L, Thurau S, van Luijk C, Seegräber M, de Bruin-Weller M. Conjunctivitis Occurring in Atopic Dermatitis Patients Treated with Dupilumab - Clinical Characteristics and Treatment. J Allergy Clin Immunol: In Practice. 2018; doi: 10.1016/j.jaip.2018.01.034.

81. Santini G, Mores N, Malerba M, Mondino C, Anzivino R, Macis $\mathrm{G}$, et al. Dupilumab for the treatment of asthma. Expert Opin Investig Drugs. 2017;26:357-66.

82. Quirce S, Phillips-Angles E, Dominguez-Ortega J, Barranco P. Biologics in the treatment of severe asthma. Allergol Immunopathol (Madr). 2017;45 Suppl 1:45-9.

\section{Ignacio Dávila}

Servicio de Alergía
Hospital Universitario de Salamanca
Edificio de Consultas
37007 Salamanca, Spain
E-mail: idg@usal.es

Servicio de Alergía

Edificio de Consultas

E-mail: idg@usal.es 